\title{
FUZZINESS MEASURE, QUANTIFIED SEMANTIC MAPPING AND INTERPOLATIVE METHOD OF APPROXIMATE REASONING IN MEDICAL EXPERT SYSTEMS
}

\author{
NGUYEN CAT HO, TRAN THAI SON, \\ TRAN DINH KHANG, AND LE XUAN VIET
}

\begin{abstract}
In the paper we shall present the applicability of hedge algebras to approximate reasoning methods. On this algebraic of viewpoint, every linguistic domain of each linguistic variable can be considered as a hedge algebra. By this we can define sensibly notions of fuzziness degree of hedges, fuzziness measure of linguistic terms and, therefrom, introduce a method for quantifying linguistic domains. The quantification of hedge algebras was introduced and investigated firstly in [16] and then developed step by step in $[12,13,19,20]$ and it is called quantified semantic mappings of hedge algebras. Here we shall present a general method for constructing flexibly quantified semantics mappings of any hedge algebras by giving fuzziness parameters and certain new ones $\theta, \alpha$ and $\beta$. In general this quantification leads to a construction of interpolative reasoning methods. Then, as an application, we construct a prototype expert system in medicine based on the new reasoning method. Experiment results seem to be appropriate to doctor diagnosis.

Tóm tắt. Trong bài báo này chúng tôi trình bày về khà năng ứng dụng của đại số gia tử vào nghiên cứu phương pháp lập luận xấp xỉ. Trên quan điêm đại số, mỗi miền ngôn ngữ của một biến ngôn ngữ có thể xem như là một đại số với cấu trúc thứ tự tự nhiên biêu thị ngữ nghĩa của ngôn ngữ. Nhờ vậy nhiều khái niệm tinh tế như độ đo tính mờ của gia từ, của các từ ngôn ngữ có thể định nghĩa rõ ràng và mang nhiều tính trực cảm. Trên cơ sở đó chúng tôi có thể đưa ra một phương pháp định lượng hoá ngữ nghĩa miền ngôn ngữ. Việc định lượng ngũ̃ nghĩa đại số gia tử được đề cập và nghiên cứu lần đầu tiên trong [16], sau đó được phát triển từng bước trong các công trình $[12,13,19,20]$ và hình thành khái niệm ánh xạ ngũ̃ nghĩa định lượng. Trong bài báo này chúng tôi còn xác lập công thức tổng quát hơn, có tính mềm dẻo, tức là có nhiều tham số tự do hơn, để tính ánh xạ ngũ nghĩa định lượng của bất kỳ đại số gia tử nào có hai phần tứ sinh. Nhờ các ánh xạ ngũ̃ nghĩa như vậy, một phương pháp lập luận xâp xỉ nội suy dễ dàng được xây dựng để giải các bài toán lập luận mờ đa điều kiện, nhiều biến. Để chứng tơ tính khả dụng của phương pháp mới chúng tôi xây dựng một hệ chuyên gia y tế thử nghiệm về bệnh viêm gan siêu vi trùng và đánh giá hiệu quả của phương pháp mới qua thử nghiệm chân đoán trên vài số liệu trong hồ sơ bệnh án thực tế.
\end{abstract}

\section{INTRODUCTION}

In $[17,18]$ we have introduced an algebraic approach to structure of linguistic domains of linguistic variables and in turn developed the theory of these algebras. In [16] a notion of metrics in these algebras is introduced and examined. Following this idea, based on the quantification of semantics of linguistic terms and hence it is called quantified semantic mapping, it is developed step by step (see $[12,13,19,20])$ in such a way that it can easily be defined by introduced notion of fuzziness degree of hedges and fuzziness measure of linguistic terms. In the paper we shall present a general method for constructing quantified semantics mappings of any linguistic domains and give a general formula to define these mappings.

So, for an arbitrary linguistic domain $\operatorname{Dom}(X)$ we can construct a quantified semantic mapping which is a one-to-one from $\operatorname{Dom}(X)$ into $[0,1]$. In [12] we showed that these mappings transform a fuzzy multiple conditional reasoning problem to a traditional interpolative problem. It makes the approximate reasoning problem more intuitive, in our opinion.

In order to prove practically the applicability of hedge algebras we shall construct an approximate reasoning algorithm for a medical expert system and use expert knowledge and data in the field of 
viral hepatitis to make experiments. The results show that the new method of approximate reasoning seems to be applicable.

\section{HEDGE ALGEBRAS: AN OVERVIEW}

In this section we shall describe generally what is a hedge algebra of a linguistic variable. In fuzzy control ones use verbal descriptions (i.e. linguistic terms) to model a dependence of one physical variable on other ones. Given a linguistic variable $X$, denote by $\operatorname{Dom}(X)$ a set of linguistic terms of $X$ called a domain of $X$. For example, if $X$ is rotation speed of a motor then $\operatorname{Dom}(X)=\{$ fast, very fast, more fast, little possibly fast, little fast, possibly fast, little slow, slow, possibly slow, very slow, more slow, very more slow, ... $\}\{\{0, W, 1\}$ is a domain of $X$. It can be considered as an algebra $A X=(\operatorname{Dom}(X), C, H, \leq)$, where $C$ is the set of generators which are the primary terms fast and slow, and the elements $W, 0$ and 1 interpreted as the neutral, the least and greatest elements in $\operatorname{Dom}(X)$; $H=\{$ very, little, possibly, more,... $\}$ is the set of hedges, which can be regarded as one-argument operations; the relation $\leq$ on $\operatorname{Dom}(X)$ is called a semantic ordering relation, because it is defined by the meaning of linguistic terms. The result of applying a hedge $h \in H$ to an element $x \in \operatorname{Dom}(X)$ is denoted by $h x$. For each $x \in \operatorname{Dom}(X)$, we denote by $H(x)$ the set of all elements $u \in \operatorname{Dom}(X)$ which are generated algebraically from $x$ by using hedges in $H$. That is $u$ can be expressed in the form $u=h_{n} \ldots h_{1} x$, where $h_{1}, \ldots, h_{n} \in H$.

In fuzzy control, this semantic ordering relation should be linear. In such case, we can intuitively order the linguistic terms which explicitly occur in the above example in a natural way. Indeed, restricting ourselves to linear hedge algebras, we can determine an ordering structure of hedge algebras, based on the following observations (a formal presentation of hedge algebras can be found in, for example, $[13,17,18])$ :

1) Each linguistic term has an intuitively semantic tendency which can be recognised by an ordering relation. Two primary terms of each linguistic variable have reverse semantic tendencies: true has a tendency of "going up" and it is called positive tendency, but false has a tendency of "going down" called negative one. These tendencies can be characterized by the ordering relationships very true > true and very false < false. For the linguistic variable $A G E$, old is positive and young is negative. From an observation of natural languages, we can find that the positivity and negativity are also identified by the inequality true $>$ false. For example, for the variable HIGH of people, tall is positive and short is negative since tall $>$ short.

2) Further, each hedge has an intuitive semantic tendency, which can be expressed also by an ordering relation. It can be observed that the one hedges increase the semantic tendency of the primary terms (called positive hedges) but the others decrease the semantic tendency of the primary terms (called negative hedges). For example, the inequalities very old $>$ old and very young $<$ young mean that very increases the semantic tendency of both terms "old" and "young" and so we say that very is positive. But, little decreases this semantic tendency and hence we say that it is negative. As a consequence, we find that two hedges $h$ and $k$ may have reverse tendencies and they are said to be converse, that is the one of which increases but the other decreases the semantic tendency of the primary terms. Also, two hedges may be compatible, that is they both either increase or decrease such semantic tendency. In the case of compatibility it may happen that one hedge is stronger than another, that is the one changes the terms more strongly than another. For example, little and possibly are compatible and little $>$ possibly, since we observe that little false $>$ possibly false $>$ false or little true $<$ possibly true $<$ true. But, it is obvious that little and very are incompatible, i.e. they are converse.

3) Moreover, as we can observe, each hedge will have an effect of increasing or decreasing semantic tendency w.r.t. any other ones. If $k$ increases the semantic tendency of a hedge $h$, we say that $k$ is positive w.r.t. $h$; and, conversely, if $k$ decreases the semantic tendency of $h$, we say that $k$ is negative w.r.t. $h$. For example, since the semantic tendency of little is expressed in the inequality little true $<$ true, it follows from the inequalities very little true $<$ little true $<$ possibly little true, that very is positive but possibly is negative w.r.t. little. Here, we find again another aspect of the universality 
of language characteristics discussed by Zadeh: the positivity or negativity of a hedge w.r.t. another one does not depend on the terms they apply to. That is if very is positive w.r.t. little then for any term $x$ we have: (if $x \leq$ little $x$ then little $x \leq$ very little $x$ ) or (if $x \geq$ little $x$ then little $x \geq$ very little $x$ ). Similarly, we observe that very is negative w.r.t. possibly and approximately, but positive w.r.t. more and very. While little is positive w.r.t. possibly, approximately and little, it is negative w.r.t. very and more.

4) An important semantic property of hedges is the so called heredity of hedges, which stems from the fact that each hedge modifies only a little but still preserves the essential meaning of each linguistic term. That is, changing the meaning of a term, it preserves the own essential meaning of this term. This means that for any hedge $h, h x$ inherits the meaning of $x$. We observe that this property can be formulated also in term of semantic ordering relation: if the meaning of $h x$ and $k x$ is expressed by $h x \leq k x$, then $h^{\prime} h x \leq k^{\prime} k x$, (i.e. $h^{\prime}$ and $k^{\prime}$ inherit, respectively, the meaning of $h x$ and $k x$ and hence they preserve the semantic ordering relationship between $h x$ and $k x$ ) and hence it implies that $H(h x) \leq H(k x)$. For example, it can be seen intuitively that from little.true $\leq$ poss.true it follows that poss.little.true $\leq$ little.poss.true, or more generally that $H$ (little.true) $\leq H$ (poss.true).

5) For any term $x$, if $h x \neq x$ then $k x \neq x$, for any hedge $k$. This property says that if the meaning of a term $x$ may be still changed properly by a hedge $h$ then it is also changed properly by any other hedge $k$. On algebraic point of view, it means that if $h x=x$ for a certain $h$ (i.e. $x$ is a fixed point of $h$ ) then $k x=x$, for all $k \in H$.

According to these observations, we can order any domains of physical linguistic variable linearly. For example, we can order the domain of the variable SPEED of a motor considered above as follows: very slow < more slow < slow < poss slow < little slow $<$ little fast $<$ little possibly fast $<$ possibly fast $<$ fast $<$ more fast $<$ very fast and so on.

Mathematically, we have the following theorems:

Theorem 2.1. (see Theorem 4 in [17]) Let the sets $H^{-}$and $H^{+}$of $A X=(\operatorname{Dom}(X), C, H, \leq)$ be linearly ordered. Then the following statements hold:

(i) For every $u \in \operatorname{Dom}(X), H(u)$ is a linearly ordered set;

(ii) If $\operatorname{Dom}(X)$ is generated from $C$ by means of hedges and $C$ is linearly ordered, then so is $\operatorname{Dom}(X)$. Moreover, if $u \leq v$ and $u$ and $v$ are independent, i.e. $u \notin H(v)$ and $v \notin H(u)$, then $H(u) \leq H(v)$.

More generally, as it is proved in [18], each domain of a linguistic variable can be axiomatised and, then, it is called a hedge algebra $A X=(\operatorname{Dom}(X), C, H, \leq)$, where $H$ is a partially ordered set of hedges, and moreover we have the following theorem for reference in sequel:

Theorem 2.2. (see [17]) Let $A X=(X, C, H, \leq)$ be a hedge algebra. Then, the following statements hold:

(i) The operations in $H^{c}$ are compatible.

(ii) If $x \in X$ is a fixed point of an operation $h$ in $H$, i.e. $h x=x$, then it is also a fixed point of the other ones.

(iii) If $x=h_{n} \ldots h_{1} u$, then there exists an index $i$ such that the suffix $h_{i} \ldots h_{1} u$ of $x$ is a canonical representation of $x$ w.r.t. $u$ (that is $x=h_{i} h_{i-1} \ldots h_{1} u$ and $h_{i} h_{i-1} \ldots h_{1} u \neq h_{i-1} \ldots h_{1} u$ ) and $h_{j} x=x$, for all $j>i$.

(iv) If $h \neq k$ and $h x=k x$ then $x$ is a fixed point.

(v) For any $h, k \in H$, if $x \leq h x(x \geq h x)$ then $x<\leq h x(x \geq>h x)$ and if $h x \leq k x, h \neq k$, then $h x<\leq k x$.

It is shown in [18] that each hedge algebra is a complete lattice with a unit element 1 and a zero element 0 and, as proved in [17], if $H$ is a chain then $A X$ is a linearly ordered set.

For convenience in the sequel, we recall here the criteria for comparing any two elements in $\operatorname{Dom}(X)$ :

Theorem 2.3. (see [18]) Let $x=h_{n} \ldots h_{1} u$ and $y=k_{m} \ldots k_{1} u$ be two arbitrary canonical representations 
of $x$ and $y$ w.r.t. $u$, respectively. Then there exists an index $j \leq \min \{m, n\}+1$ such that $h_{j^{\prime}}=k_{j^{\prime}}$, for all $j^{\prime}<j$ (here as a convention it is understood that if $j=\min \{m, n\}+1$, then either $h_{j}=I$ for $j=n+1 \leq m$ or $k_{j}=I$ for $\left.j=m+1 \leq n\right)$ and

(1) $x=y$ iff $m=n$ and $h_{j} x_{j}=k_{j} x_{j}$;

(2) $x<y$ iff $h_{j} x_{j}<k_{j} x_{j}$;

(3) $x$ and $y$ are incomparable iff $h_{j} x_{j}$ and $k_{j} x_{j}$ are incomparable.

\section{FUZZINESS MEASURE OF LINGUISTIC TERMS}

Fuzziness degree is a concept which is not easy to determine intuitively and hence it is very difficult to define in framework of the fuzzy sets theory. In this section we shall shown that hedge algebras can be used as a basis for defining a fuzziness degree of terms in a reasonable and obvious way.

First of all, let us consider the following observation. It can be argued that the more specific a term is, the less fuzziness degree it is. For example, the fuzziness degree of the terms 'more or less true' (denoted by MLtrue for short), 'possibly true' is less than that of the term 'true'. Let the meaning of linguistic terms be represented by fuzzy sets. One notion of fuzziness degree is the so-called fuzziness index which is defined by the relative distance between the fuzzy set representing this term and its nearest crisp set (see [1]). It seems to be appropriate to our intuition because the fuzziness index of crisp set is equal to zero. However, if we represent the term 'true' by the fuzzy set $\mu_{\text {true }}(t)=t$ on the unit interval $[0,1]$, and 'MLtrue' by $\mu_{M L t r u e}(t)=t^{\alpha}$ with $\alpha=2 / 3<1$, then the fuzziness index of 'true' equals to $1 / 4$, but the fuzziness index of ' $M$ Ltrue' equals to

$$
\frac{4-\sqrt{2}}{10}>\frac{1}{4}
$$

which is obviously not appropriate to our requirement above.

Therefore, it will be more convenient to find out firstly some general intuitive properties of fuzziness degree of linguistic terms. These properties will form an important basic for establishing a suitable definition of fuzziness degree.

Let us denote by fus $(\tau)$ the fuzziness degree of a term $\tau$ in a domain $\operatorname{Dom}(X)$ of a linguistic variable $X$, that is assumed to take values in $[0,1]$. It can be argued that fus $(\tau)$ is necessary to be satisfied the following intuitive properties:

(1) fus $(\tau)=0$, for any non-vague value $\tau$.

(2) If $h$ is a hedge and $\tau$ is a vague value, then $h \tau$ is more specific than $\tau$ and hence we should have fus $(h \tau) \leq$ fus $(\tau)$.

(3) The following property may be more difficult to be recognised. Let us take into consideration two vague terms 'true' and 'false' which are the generators of a hedge algebra. Because these concepts are contradictory, i.e. they are reverse and complementary, we can adopt the following condition:

$$
\text { fus }(\text { true })+\text { fus }(\text { false }) \leq 1 .
$$

We find that if fus $($ true $)+$ fus $($ false $)<1$, then there must be still a vague term $\tau$ different from and complementary to the values 'true' and 'false' so that fus (true $)+$ fus $(f a l s e)+$ fus $(\tau)=1$. It is not the case in any natural languages, and therefore we should have fus $($ true $)+$ fus $($ false $)$ $=1$. Therefore, if $c^{+}$and $c^{-}$are the only primary terms of $\operatorname{Dom}(X)$, then we always have

$$
f u s\left(c^{+}\right)+f u s\left(c^{-}\right)=1 .
$$

(4) Now consider a system of hedges $H=\{$ Very, More, Possibly, Little $\}$ and a set of vague values H/true $\}=\{$ Very true, More true, Possibly true, Little true $\}$, whose elements are more specific than the term 'true' which the hedges apply to. By point (2) the fuzziness degree of 'true' is greater than the one of every term in H[true]. But, how does the fuzziness of 'true' take shape in 
our mind? We may imagine intuitively that the fuzziness of 'true' are formed by the meaning of all terms which still express an aspect of the meaning of 'true'. Therefore, all "what" form the fuzziness of every term in the set H[true], that are obtained by modifying the term 'true', will also contribute to form the fuzziness of 'true'. Moreover, since each hedge $h$ has its own meaning: which is different from the other ones $k$ in $H$, we can argue that "what" which participates in forming the fuzziness of one term $x$ in $H(h$ true $)$ can not participate in forming another term $y$ in $H(k$ true $)$. So, analogously as independent events in the probabilistic theory, we can adopt the following condition:

$$
\text { fus }(\text { Very.true })+\text { fus }(\text { More.true })+\text { fus }(\text { Poss.true })+\text { fus }(\text { Littletrue }) \leq \text { fus(true }) \text {, }
$$

and if $H$ is the set of all hedges under consideration then, similarly as the argument in point (3) above, we should have

$$
\text { fus }(\text { Verytrue })+\text { fus }(\text { Moretrue })+\text { fus }(\text { Possiblytrue })+\text { fus }(\text { Littletrue })=\text { fus }(\text { true }) .
$$

In general case, for any term $\tau$, we have

$$
\text { fus }(\operatorname{Very} \tau)+\text { fus }(\text { More } \tau)+\text { fus }(\text { Possibly } \tau)+\text { fus }(\text { Little } \tau)=\text { fus }(\tau) \text {. }
$$

In Figure 1 we give an example of fuzziness measure on $\operatorname{Dom}(T R U T H)$.

Now, we shall show that based on hedge algebras it will be easy to define fuzziness measure on linguistic domains of a linguistic variable.

Let us consider a linguistic domain $\operatorname{Dom}(X)$ which is considered as a hedge algebra $A X=$ $(\operatorname{Dom}(X), C, H, \leq)$.

Definition 3.1. Let us consider a hedge algebra of a linguistic $X, A X=(\operatorname{Dom}(X), C, H, \leq)$. A function $\varphi: \operatorname{Dom}(X) \rightarrow[0,1]$ is said to be a fuzziness measure on $\operatorname{Dom}(X)$ provided that there exists a probability $P$ on $\operatorname{Dom}(X)$ such that $P$ is defined on all sets of the form $H(\tau)$, for every term $\tau$ in $\operatorname{Dom}(X)$, and $P(H(\tau))=0$ when and only when $\tau \in\{0, W, 1\}$ and $\varphi(\tau)=$ $P(H(\tau))$.

So, the "size" of set $H(\tau)$ describes fuzziness degree of the term $\tau$ and hence the measure of the set $H(\tau)$ will expresses the fuzziness measure of $\tau$.

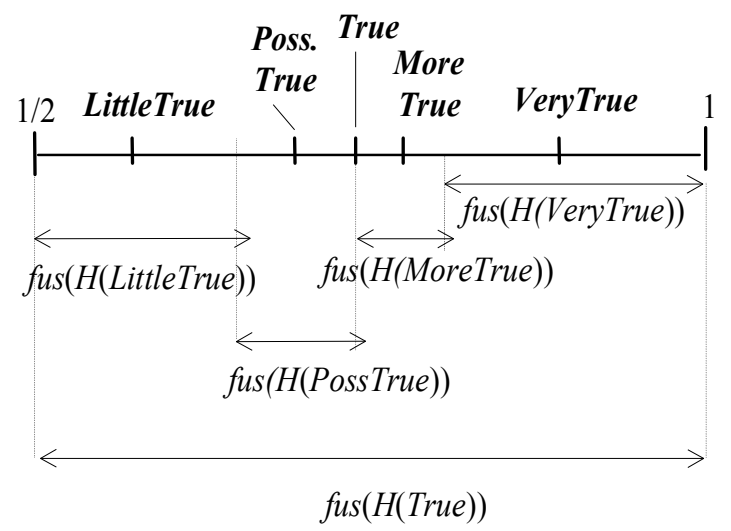

We can point out that $\varphi$ satisfies all intuitive properties presented above:

Property (1) is evident by the definition of $\varphi$. Property (2) is derived from the fact that $H(h \tau) \subseteq$ $H(\tau)$. Since $H\left(c^{+}\right) \cup H\left(c^{-}\right) \cup\{0, W, 1\}=\operatorname{Dom}(X)$, Property (3) follows. Analogously, Property (4) follows from the equalities $\cup\{H(h \tau): h \in H\}=H(\tau)$ and $H(h \tau) \cap H\left(h^{\prime} \tau\right)=\emptyset$, for any $h \neq h^{\prime}$.

For convenience, we list here again some properties of fuzziness measure:

Property (p1): $\varphi(0)=\varphi(W)=\varphi(1)=0$.

Property (p2): $\varphi(h \tau) \leq \varphi(\tau)$, for any term $\tau$ and any hedge $h \in H$.

Property (p3): $\varphi\left(c^{+}\right)+\varphi\left(c^{-}\right)=1$, where $c^{+}$and $c^{-}$are the only primary terms of $\operatorname{Dom}(X)$.

Property (p4): $\left\{\sum\{\varphi(h \tau): h \in H\}=\varphi(\tau)\right.$, for any term $\tau$ in $\operatorname{Dom}(X)$.

We can rewrite Property $(\mathrm{p} 4)$ as follows:

$$
\sum\{\varphi(h \tau) / \varphi(\tau): h \in H\}=1,
$$

i.e. this sum is invariant when term $\tau$ runs in the whole domain $\operatorname{Dom}(X)$. It may also be appropriate to our intuition to assume that the ratio $\varphi(h \tau) / \varphi(\tau)$ is also constant when $\tau$ runs in the whole domain 
$\operatorname{Dom}(X)$. This ratio characterises the fuzziness degree of each hedge $h \in H$.

From now on we always assume that fuzziness measure $\varphi$ satisfies the following property:

Property (p5): The ratio $\varphi(h \tau) / \varphi(\tau)$ does not depend on term $\tau$ and hence it is called, for uniformity, the fuzziness measure of $h$ and denoted by $\mu(h)$.

Theorem 3.1. Fuzziness measure on $\operatorname{Dom}(X)$ is uniquely determined by the parameters $\varphi\left(c^{+}\right), \varphi\left(c^{-}\right)$ and $\mu(h), h \in H$, which satisfy the equalities $\varphi\left(c^{+}\right)+\varphi\left(c^{-}\right)=1, \sum\{\mu(h): h \in H\}=1$, and $\varphi(\tau)$ is defined recursively by $\varphi\left(h x^{\prime}\right)=\mu(h) \varphi\left(x^{\prime}\right)$, for any term $x=h x^{\prime}, h \in H$.

\section{QUANTIFYING LINGUSTIC DOMAINS OF A LINGUSTIC VARIABLE}

A most important characteristic of linguistic terms is qualitative. It is a human powerful manner for formulating experts knowledge. However, in computational approach to human reasoning, especially in fuzzy control we need quantitative characteristic. Therefore, it arises a natural requirement to quantify fuzzy data or, in general, linguistic terms.

In our approach, we do not use fuzzy sets to interpret the meaning of vague concepts, but just these concepts as being elements in the structure of a hedge algebra, i.e. in $\operatorname{Dom}(X)$. In this case in order to quantify linguistic terms, we establish a suitable mapping from $\operatorname{Dom}(X)$ into the unit interval $[0,1]$. By its meaning we call it quantified semantic mapping.

On account of the above examination, we have a reasonable way to construct quantified semantic mappings on a given linguistic domain.

Let us consider a hedge algebra $A X=(\operatorname{Dom}(X), C, H, \leq)$, where $H=H^{-} \cup H^{+}$, and suppose that $H^{-}=\left\{h_{-1}, h_{-2}, \ldots, h_{-q}\right\}$, where $h_{-1}<h_{-2}<\ldots<h_{-q}$, and $H^{+}=\left\{h_{1}, \ldots, h_{p}\right\}$, where $h_{1}<\ldots<h_{p}$ and $h_{0}=I$.

First, we need a notation.

Definition 4.1. (Sign function). Function Sign: $X \rightarrow\{-1,0,1\}$ is a mapping defined recursively as follows, where the hedges $\mathrm{h}$ and $\mathrm{h}$ ' are arbitrary:

a) $\operatorname{Sign}\left(c^{-}\right)=-1$, and $\operatorname{Sign}\left(h c^{-}\right)=+\operatorname{Sign}\left(c^{-}\right)$, if $h c^{-}<c^{-}$(i.e. if $h$ is positive w.r.t. $c^{-}$;)

$\operatorname{Sign}\left(h c^{-}\right)=-\operatorname{Sign}\left(c^{-}\right)$, if $h c^{-}<c^{-}$(i.e. if $h$ is negative w.r.t. $c^{-}$;)

$\operatorname{Sign}\left(c^{+}\right)=+1$, and $\operatorname{Sign}\left(h c^{+}\right)=+\operatorname{Sign}\left(c^{+}\right)$, if $h c^{+}<c^{+}$(i.e. if h is positive w.r.t. c+;)

$\operatorname{Sign}\left(h c^{+}\right)=-\operatorname{Sign}\left(c^{+}\right)$, if $h c^{+}<c^{+}$(i.e. if h isnegative w.r.t. $c^{+}$;)

b) $\operatorname{Sign}\left(h^{\prime} h x\right)=-\operatorname{Sign}(h x)$ if $h^{\prime}$ is negative w.r.t. $h$ and $h^{\prime} h x \neq h x$,

c) $\operatorname{Sign}\left(h^{\prime} h x\right)=\operatorname{Sign}(h x)$ if $h^{\prime}$ is positive w.r.t. $h$ and $h^{\prime} h \neq h x$.

d) $\operatorname{Sign}\left(h^{\prime} h x\right)=0$ if $h^{\prime} h x=h x$.

Proposition 4.1. For any hedge $h$ and element $x$, if $\operatorname{Sign}(h x)=+1$ then $h x>x$, and if $\operatorname{Sign}(h x)=$ -1 then $h x<x$

Definition 4.2. Let the parameters $\varphi\left(c^{+}\right), \varphi\left(c^{-}\right)$and $\mu(h), h \in H$ be given such that $\varphi\left(c^{+}\right)+\varphi\left(c^{-}\right)=$ 1, $\sum\{\mu(h): h \in H\}=1$. A quantified semantic mapping $\nu$ on $\operatorname{Dom}(X)$ is defined as follows:

a) $\nu(W)=\theta=\varphi\left(c^{-}\right), \nu\left(c^{-}\right)=\theta-\alpha \varphi\left(c^{-}\right), \quad \nu\left(c^{+}\right)=\theta+\alpha \varphi\left(c^{+}\right)$;

b) $\nu\left(h_{j} x\right)=\nu(x)+\operatorname{Sign}\left(h_{j} x\right)\left\{\sum_{i=1}^{j} \varphi\left(h_{i} x\right)-\omega\left(h_{j} x\right) \varphi\left(h_{j} x\right)\right\}$, for $1 \leq j \leq p$, and

$$
\nu\left(h_{j} x\right)=\nu(x)+\operatorname{Sign}\left(h_{j} x\right)\left\{\sum_{i=-1}^{j} \varphi\left(h_{i} x\right)-\omega\left(h_{j} x\right) \varphi\left(h_{j} x\right)\right\}, \text { for }-q \leq j \leq-1,
$$

that we can write in one formula as follows:

$$
v\left(h_{j} x\right)=\nu(x)+\operatorname{Sign}\left(h_{j} x\right)\left\{\sum_{i=\operatorname{Sign}(j)}^{j} \varphi\left(h_{i} x\right)-\omega\left(h_{j} x\right) \varphi\left(h_{j} x\right)\right\}, \text { for } j \in[-q \wedge p],
$$

where $[-q \wedge p]$ denotes the set of all $j$ such that $-q \leq j \leq p$ and $j \neq 0, \varphi(x)$ is defined as in Theorem 3.1 and:

$$
\omega\left(h_{j} x\right)=\frac{1}{2}\left[1+\operatorname{Sign}\left(h_{j} x\right) \operatorname{Sign}\left(h_{p}, h_{j} x\right)(\beta-\alpha)\right] \in\{\alpha, \beta\} .
$$




\section{Proposition 4.2.}

(i) For all $x \in X, 0 \leq \nu(x) \leq 1$.

(ii) For all $x, y \in X, x<y$ implies $v(x)<v(y)$.

To illustrate our method of constructing quantified semantic mapping we give an example.

Now we give some examples of computing some values of the quantified semantic mapping $\nu$.

+ For $x=c^{-}=$Small, from Definition 4.1 we have $\nu($ small $)=\theta-\alpha f m($ small $)=0.5-0.5 \times 0.5=$ 0.25 .

+ For $x=$ VerySmall, we have $j=p=2, \operatorname{Sign}\left(h_{2}\right.$ Small $)=-1, \operatorname{Sign}\left(h_{2} h_{j}\right.$ Small $)=$ $\operatorname{Sign}\left(h_{2} h_{2}\right.$ Small $)=-1$ and $\omega\left(h_{p}\right.$ Small $)=\frac{1}{2}[1+(-1)(-1)(\beta-\alpha)]=0.5$ and $\nu($ VerySmall $)=\nu($ Small $)+(-1)\left\{f m\left(h_{1}\right.\right.$ Small $)+f m\left(h_{2}\right.$ Small $)-0.5 f m\left(h_{2}\right.$ Small $\left.)\right\}=$ $\nu($ Small $)+(-1)\left\{\mu(h 1) f m(\right.$ Small $)+0.5 \mu\left(h_{2}\right) f m($ Small $\left.)\right\}=$ $0.25-\{0.10 \times 0.5+0.5 \times 0.40 \times 0.5\}=0.10$.

+ For $x=$ LittleVerySmall, we have $j=-q=-2, \operatorname{Sign}\left(h_{-2}\right.$ VerySmall $)=+1$, $\operatorname{Sign}\left(h_{2} h_{-2}\right.$ VerySmall $)=+1$ and $\omega\left(h_{-2}\right.$ VerySmall $)=0.5$. Hence, $\nu\left(h_{-2}\right.$ VerySmall $)=$ $\nu($ VerySmall $)+(+1)\left\{f m\left(h_{-1}\right.\right.$ VerySmall $)+f m\left(h_{-2}\right.$ VerySmall $)-0.5 f m\left(h_{-2}\right.$ VerySmall $\left.)\right\}=$ $\nu($ VerySmall $)+\{\mu($ Possibly $) \mu($ Very $) \times f m($ small $)+0.5 \mu($ Little $) \mu($ very $) f m($ small $)\}=$ $0.10+\{0.10 \times 0.40 \times 0.5+0.5 \times 0.40 \times 0.40 \times 0.5\}=0.10+0.06=0.16$.

The other values of the quantified semantic mapping $\nu$ are computed in a similar way and the results are given in Table 1.

For $\theta=0.6, \mu($ Less $)=0.35, \mu($ Possible $)=0.25, \mu($ More $)=0.15$ and $\mu($ Very $)=0.25$, the values of the mapping $\nu$ are given in Table 2 .

\begin{tabular}{|} 
Table 1. $\mu($ Little $)=0.40, \mu($ possible $)=0.10, \mu($ more $)=0.10, \mu($ very $)=0.40, \theta=0.5$ \\
\hline Very Very Small & 0.040000 & Very Less Large & 0.540000 \\
More Very Small & 0.090000 & More Less Large & 0.590000 \\
Very Small & 0.100000 & Less Large & 0.600000 \\
Possible Very Small & 0.110000 & Possible Less Large & 0.610000 \\
Less Very Small & 0.160000 & Less Less Large & 0.659999 \\
Very More Small & 0.210000 & Less Possible Large & 0.710000 \\
More More Small & 0.222500 & Possible Possible Large & 0.722500 \\
More Small & 0.225000 & Possible Large & 0.725000 \\
Possible More Small & 0.227500 & More Possible Large & 0.727499 \\
Less More Small & 0.240000 & Very Possible Large & 0.740000 \\
Small & 0.250000 & Large & 0.750000 \\
Very Possible Small & 0.260000 & Less More Large & 0.760000 \\
More Possible Small & 0.272500 & Possible More Large & 0.772500 \\
Possible Small & 0.275000 & More Large & 0.775000 \\
Possible Possible Small & 0.277500 & More More Large & 0.777500 \\
Less Possible Small & 0.290000 & Very More Large & 0.790000 \\
Less Less Small & 0.340000 & Less Very Large & 0.840000 \\
Possible Less Small & 0.390000 & Possible Very Large & 0.890000 \\
Less Small & 0.400000 & Very Large & 0.900000 \\
More Less Small & 0.410000 & More Very Large & 0.910000 \\
Very Less Small & 0.460000 & Very Very Large & 0.960000 \\
\hline
\end{tabular}


Table 2. $\theta=0.6, \mu($ Less $)=0.35, \mu($ possible $)=0.25, \mu($ more $)=0.15, \mu($ very $)=0.25$

\begin{tabular}{|l|l|l|l|}
\hline Very Very Small & 0.015 & Very Less Large & 0.613999 \\
More Very Small & 0.0465 & More Less Large & 0.6434 \\
Very Small & 0.06 & Less Large & 0.655999 \\
Possible Very Small & 0.075 & Possible Less Large & 0.669999 \\
Less Very Small & 0.129 & Less Less Large & 0.720399 \\
Very More Small & 0.159 & Less Possible Large & 0.753999 \\
More More Small & 0.1779 & Possible Possible Large & 0.789999 \\
More Small & 0.186 & Possible Large & 0.799999 \\
Possible More Small & 0.195 & More Possible Large & 0.808999 \\
Less More Small & 0.2274 & Very Possible Large & 0.83 \\
Small & 0.24 & Large & 0.84 \\
Very Possible Small & 0.255 & Less More Large & 0.8484 \\
More Possible Small & 0.2865 & Possible More Large & 0.87 \\
Possible Small & 0.3 & More Large & 0.876 \\
Possible Possible Small & 0.315 & More More Large & 0.8814 \\
Less Possible Small & 0.369 & Very More Large & 0.894 \\
Less Less Small & 0.4194 & Less Very Large & 0.913999 \\
Possible Less Small & 0.495 & Possible Very Large & 0.95 \\
Less Small & 0.516 & Very Large & 0.96 \\
More Less Small & 0.5349 & More Very Large & 0.969 \\
Very Less Small & 0.579 & Very Very Large & 0.99 \\
\hline
\end{tabular}

\section{INTERPOLATIVE REASONING METHOD}

In fuzzy control, we often deal with multiple conditional fuzzy reasoning problems, the physical variables of which are normally modelled by linguistic variables with real domains usually being linearly ordered sets. So, hedge algebras as models of physical variable must be linearly ordered sets as well. This suggests us in this section to deal with a new interpolation reasoning method to solve multiple conditional fuzzy reasoning problem, based on quantified semantic mappings examined above.

Consider a fuzzy model:

If $X_{1}=A_{11}$ and $\ldots$ and $X_{m}=A_{1 m}$ then $Y=B_{1}$

If $X_{1}=A_{21}$ and $\ldots$ and $X_{m}=A_{2 m}$ then $Y=B_{2}$

If $X_{1}=A_{n 1}$ and $\ldots$ and $X_{m}=A_{n m}$ then $Y=B_{n}$

where $A_{i j}$ and $B_{i}, i=1,2, \ldots, n$ and $j=1,2, \ldots, m$ are verbal descriptions of physical variables $X_{j}$ and $Y$, respectively.

Using fuzzy sets-based methods in fuzzy multiple conditional reasoning, we should carry out the following main steps:

1) To determine an appropriate reasoning method: One may choose a method based on composition rule (called also generalised Modus Ponens (see $[4,5,9,10,25]$ ) or fuzzy interpolation reasoning methods (see $[6,7,24,26,27,28]$ ). Note that their efficiency depends on a number of factors such as implication operators, composition operators, aggregation operators, and so on. 
2) To determine fuzzy sets, i.e. membership functions: these functions should suitably represent the meaning of linguistic terms occurring in the fuzzy model and in fuzzy input data, based on experts experiences and/or practical experiments.

3) To transform (in fuzzy control) the outputs of the method, which in general are also fuzzy sets, into real values by a defuzzification method.

It can be seen, in authors' opinion, that using these methods ones lose intuition and meet with many difficulties to recognise their behavior, since the results depend on several factors, whose influences on the chosen method can not be evaluated.

Here we introduce a more intuitive approach, which bases on interpolation reasoning methods. The idea is simply as follows: to solve a multiple conditional fuzzy reasoning problem with fuzzy model given by equation (5.1), we interpret each if-then statement as defining a point and, therefore, this model defines a fuzzy curve $C_{f}$ in the Cartesian product $\operatorname{Dom}\left(X_{1}\right) \times \ldots \times \operatorname{Dom}\left(X_{m}\right) \times \operatorname{Dom}(Y)$, where $\operatorname{Dom}\left(X_{i}\right)$ and $\operatorname{Dom}(Y)$ are linguistic domains considered as hedge algebras of $X_{i}$ and $Y$, respectively. Then, the fuzzy reasoning problem "For a given fuzzy model (5.1) and an input $A=\left(A_{1}, A_{2}, \ldots, A_{m}\right)$ find an output $B$ corresponding to $A$ " may be understood as an interpolation problem for the fuzzy curve $C_{f}$ in $\operatorname{Dom}\left(X_{1}\right) \times \ldots \times \operatorname{Dom}\left(X_{m}\right) \times \operatorname{Dom}(Y)$.

The main steps of our method are simply as follows:

1) To construct quantified semantic mappings $\nu_{X_{i}}$ and $\nu_{Y}$, which map the hedge algebras of $X_{i}$ and $Y$ into the unit interval $[0,1]$, respectively. As examined above, these mappings are determined by the fuzziness measure of primary terms and of linguistic hedges, which can be considered as users parameters to adapt specific applications.

2) Under mappings $\nu_{X_{i}}$ and $\nu_{Y}$, linguistic values will be transformed into real values in $[0,1]$ and, hence, we can establish a transformation which transforms the fuzzy curve $C_{f}$ in $\operatorname{Dom}\left(X_{1}\right) \times$ $\ldots \times \operatorname{Dom}\left(X_{m}\right) \times \operatorname{Dom}(Y)$ into a real curve $C_{r, m+1}$ in $\left[0, d_{1}\right] \times\left[0, d_{2}\right] \times \ldots \times\left[0, d_{m}\right] \times[0, b]$, where $\left[0, d_{i}\right]$ and $[0, b]$ are the domains of the basic variables of $X_{i}$ and $Y$, respectively.

3) To transform the real curve $C_{r, m+1}$ in step 2) into a real curve $C_{r, 2}$ in $[0, a] \times[0, b]$ by using an aggregation operator $\alpha$ (see [21,22]) as follows:

+ The value $a$ of $[0, a]$ is calculated by $a=a\left(d_{1}, d_{2}, \ldots, d_{m}\right)$.

+ For each index $i, a_{i j}=\nu_{X_{j}}\left(A_{i j}\right)$ for $j=1,2, \ldots, m$, we determine a point $\left(a_{i}, b_{i}\right)$ of $C_{r, 2}$ by the following equations:

$$
a_{i}=\alpha\left(a_{i 1}, a_{i 2}, \ldots, a_{i m}\right) \text { and } b_{i}=\nu_{Y}\left(B_{i}\right) .
$$

Use the classical linear interpolative method to compute the output corresponding to an input data $a_{0}=\alpha\left(\nu_{X_{1}}\left(A_{01}\right), \nu_{X_{2}}\left(A_{02}\right), \ldots, \nu_{X_{m}}\left(A_{0 m}\right)\right)$, for the given input terms $X_{1}=A_{01}, X_{2}=$ $A_{02}, \ldots, X_{m}=A_{0 m}$.

\section{AN APPLICATION OF THE NEW METHOD IN CONSTRUCTION OF A REASONING ALGORITHM IN MEDICINE}

To illustrate the applicability of the new method we constructed an experiment system for diagnosing viral hepatitis. We collected about 200 archives of viral hepatitis patients in Army Central Hospital at Hanoi and discussed with a few high experienced experts in this medicine area to build up a knowledge base consisting about 70 rules which are formulated in terms of linguistic values. Normally, as it can be observed, each rule has a truth degree which is expressed also by linguistic term of TRUTH.

An example of this rule is the following:

IF "yellow colour skin symptom" is "obvious"

AND "temperature" is "high"

AND "gall status" is "achy"

THEN "gall blocked symptom" is "possible";

WITH Truth belief degree $:=\tau$, where $\tau \in \mathcal{T}$ - the set of all linguistic terms of TRUTH such as "true" and all values $t \in[0,1]$. 
In social phenomena, we are most often dealing with sentences containing vague concepts associated with a truth degree $\tau \in \mathcal{T}$ and called linguistically fuzzy assertions (LF-assertion, for short). So, we shall formalise such an assertion by a pair $\langle F, \tau\rangle$, where $F$ is a linguistically fuzzy sentence (LF-sentence, for short), and $\tau \in \mathcal{T}$.

For LF-sentences we can define roughly as follows. Firstly, by elementary LF-sentences we mean those which could be called LF-predicates. Examples of such sentences are the following: 'Robert is very old', 'The gas gauge of $z$ reads normal', 'Part $X$ of the engine $e$ depends on part $Y$ strongly' and 'The motor $m$ turns well'. Because we want deal with vague concepts, they can be separated from the remaining terms and therefore LF-predicates will be divided into two parts. The first one is those that build the main meaning of such sentences. It consists of all terms excepts vague concepts occurring in the sentences. They can be considered as ordinary predicates and called the substance part. Examples for it are '(the age of) Robert is' in the first sentence above, 'The gas gauge of $z$ reads' in the second, 'Part $X$ of the engine $e$ depends on part $Y$ ' in the third and 'The motor turns' in the forth.

The second part of LF-predicate consists of the remaining terms which are vague concepts. It is called the value part. In the above sentences they are 'very old', 'normal', 'strongly' and 'well', respectively. It can be seen that the substance part determines the possible vague terms which are regarded as its values. Therefore, the substance part plays a role which is very similar to that of linguistic variable. Hence, an LF-predicate can be denoted by a pair $(p, u)$, where $p$ is a (classical) predicate of $n$ arguments and $u$ is a vague concept. With this notation, the above examples can be written by (AGE(Robert),very-old), (READ.GAS-GAUGE(z), normally), (DEPEND (part (e) $\left._{\text {, part }}(e)\right)$, strongly) and (TURN.MOTOR $(m)$, well), respectively, where Robert is individual constant, $z$, $\operatorname{part}_{X}(e), \operatorname{part}_{X}(e)$ and $m$ are individual variables.

Secondly, composed LF-sentences are formed recursively from elementary ones by means of logical connectives such as 'and', 'or', 'if-then' and 'not', which are denoted correspondingly by $\wedge, \vee, \rightarrow$, and $\neg$ and called conjunction, disjunction, implication and negation, respectively. An example for composed LF-sentences is 'if a person $x$ is old then he or she can not run quickly' and this sentence can be expressed by $(A G E(x$, old $) \rightarrow \neg(R U N(x)$, quickly $)$.

By $\mathcal{F}$ we denote the set of all LF-sentences.

In the paper we consider a set $\mathcal{R}$ of assertions of the form $\langle F \rightarrow P, \tau\rangle$, called a LF-rule. The problem of reasoning is that how can we deduce certain conclusions from a given knowledge $\mathcal{R}$ and a set $\mathcal{H}$ of input data?

Next we shall build a reasoning mechanism to answer the following question: Given a set $\mathcal{C}$ of conclusions of the form $\langle\mathcal{Q}, \tau\rangle$, whether $\mathcal{C}$ can be deduced from $\mathcal{R}$ and $\mathcal{H}$ ?

Our reasoning algorithm consists of the following main procedures:

Let a set $\mathcal{R}$ of LF-rules, a set $\mathcal{H}$ of assertions and a set of possible conclusions $\mathcal{C}$ be given.

\section{1) Variable names}

+ MIDCONS: This variable is used to denote the set consisting of all facts in $\mathcal{H}$ and the concluded facts which can be deduced from $\mathcal{R}$ and $\mathcal{H}$ up to a certain point of running time of the reasoning algorithm.

+ RULE: It is used to denote the set of rules in $\mathcal{R}$ which are still unused by the reasoning algorithm up to a point of its running time.

+ SAT: Denote the set of rules in $\mathcal{R}$ which are applicable to the data in MIDCONS.

+ MODEL: Denote a set of compatible rules in $\mathcal{R}$ which forms a fuzzy model for a multiple conditional fuzzy reasoning method, where we mean that two rules are compatible if their linguistic variables in the if-part and in the then-part are respectively the same.

+ INPUT: Denote an input data for the fuzzy model defined in MODEL. It will be an input of the procedure prITPLREAS described below.

+ CHOUT: Contains a rule which is chosen from SAT w.r.t. a criterion given below. 
+ TRACE: It is used to display the way of reaching the result. It contains a list of sequent rules in $\mathcal{R}$ which are used by the reasoning algorithm.

+ ITPLREAS: Contains an output result in the form of LF-sentence produced by a linguistic interpolation reasoning procedure prITPLREAS presented below.

2) Procedures

a) Rough Sieve Procedure: prSIEV E(RULE, MIDCONS)

The procedure fulfills a function that eliminates those rules in $R U L E$ which are certainly not applicable to data in MIDCONS. Here applicability to MIDCONS is understood as follows: a rule $r$ is said to be applicable to MIDCONS if all linguistic variables which occur in $r$ must also occur in $M I D C O N S$. The results are put in $S A T$.

b) Procedures prCHOOSE(SAT, criteria) :

It chooses a rule in $S A T$ which satisfies a designed criterion for the sequential step of reasoning. Below we shall introduce a decision criterion, denoted by criteria, based on combining some heuristic indications for choosing a better LF-rule in each step of reasoning. The output is put in CHOUT.

c) Procedure prMODEL $(S A T, C H O U T)$ :

Firstly, two rules are said to be compatible if they have the same linguistic variables in if-part as well as in then-part. This procedure establish a fuzzy model in a form of (5.1) by taking all rules in $S A T$ which are compatible with the rule in CHOUT. The outputs are put in MODEL.

d) Procedure prINTPUT(MODEL, MIDCONS) :

The procedure produces an input data from data in MIDCONS for the linguistic interpolation reasoning procedure prITPLREAS presented in the next. It takes linguistic data with greatest value whose linguistic variables occurring in if-part of the sentences in $M O D E L$. The largest values guarantee that results of the next procedure is of high linguistic belief degree. The output will be put in $I N P U T$.

e) Procedure prITPLREAS(MODEL, $\nu, \alpha$, INPUT):

Here for each linguistic variable $X$ we denote, as above, by $\nu_{X}$ a quantified semantic mapping of $X$. Put $\nu=\left\{\nu_{Y}, v_{X_{i}}: X_{i}\right.$ is a linguistic variable occurring in the if-part of the rules in the fuzzy model given in $M O D E L$ and $Y$ is the unique variable in the then-part $\}$ and $\alpha$ is an aggregation operator. Suppose $\nu, \alpha$ are given. So, this procedure has the following functions:

+ For each input data given in INPUT it computes the corresponding value $b$ of $\nu_{Y}$ by using interpolative reasoning method presented in Section 5.

+ Let $k$ be the greatest length of the terms which occur in the then-parts of the fuzzy model given in $M O D E L$. For each has been computed value $b$ of the linguistic variable $Y$, it determines a term $\tau_{Y}$ in $\operatorname{Dom}(Y)$ of the length not greater than $k+2$ such that $\left|b-\nu_{Y}\left(\tau_{Y}\right)\right|$ is smallest.

+ Put the result in a form of LF-sentence $\left(P_{Y}\left(y, \tau_{Y}\right)\right)$ in $\operatorname{ITPLREAS}$, where $P_{Y}\left(y, \tau_{Y}\right)$ is the predicate of $Y$ and $y$ is an individual variable.

3) Linguistic reasoning algorithm LR-ALGORITHM

LR-ALGORITHM

Input: $\mathcal{R}, H, C, \nu, \alpha$;

\{

$R U L E:=\mathcal{R}$

$M I D C O N S:=\mathcal{H}$

$T R A C E:=\phi ;$ (the empty trace)

$S A T:=\operatorname{prSIEVE}(R U L E, M I D C O N S)$;

If $(\mathcal{C} \subseteq M I D C O N S)$ then exit("Success");

While $(S A T \neq \emptyset)$ do

\{

CHOUT:=prCHOOSE(SAT, criteria);

MODEL := prMODEL $(S A T, C H O U T)$;

$I N P U T:=\operatorname{prINPUT}(M O D E L, M I D C O N S) ;$ 


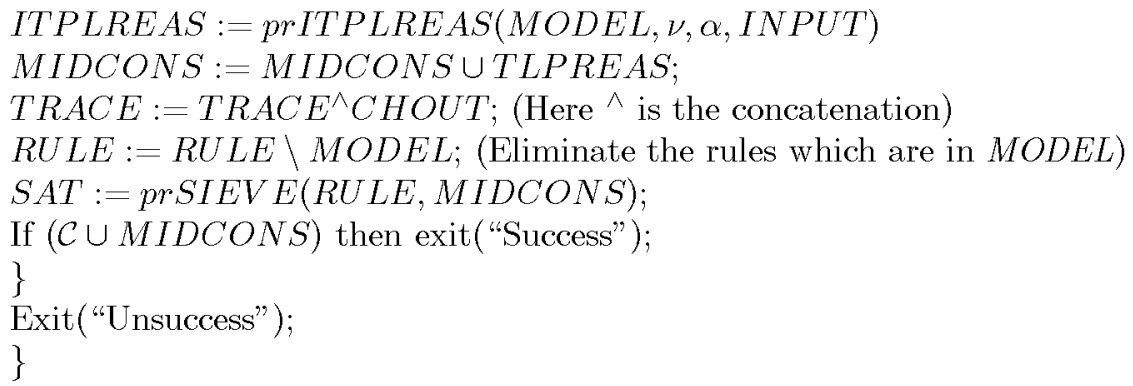

\section{4) Heuristic criteria for prCHOOSE}

Now we introduce heuristic criteria for choosing a rule which is "better" than the other ones at each step of reasoning.

(H1) CriterionH1 : A rule $r \in S A T$ of the form "If $X_{1}=A_{1}$ and $X_{2}=A_{2}$ and... and $X_{m}=A_{m}$ then $Y=B$ " will be of high choosing possibility if the difference between their quantified semantic values and the corresponding ones of LF-sentences $A_{01}, A_{02}, \ldots, A_{0 m}$ in $M I D C O N S$ is small. Put

$h_{1}(r)=1-\max \left\{\left|\nu_{X_{i}}\left(A_{2}\right)-\nu_{X_{i}}\left(A_{02}\right)\right|: i=1,2, \ldots, m\right\}$.

(H2) CriterionH2 : A rule $r \in S A T$ will be of high choosing possibility if its quantified truth degree $t(r)=\nu($ truth degree of $r)$ is high. So, we put $h_{2}(r)=t(r), r \in S A T$.

(H3) CriterionH3 : The possibility of a rule $r$ generating a new linguistic variable:

$$
h_{3}(r)= \begin{cases}1 & \text { if } r \text { generates a new linguistic variable; } \\ 0 & \text { if not. }\end{cases}
$$

(H4) Criterion $H 4$ : The possibility of a rule $r$ generating more applicable rules: Suppose at the moment $k$ we have a set of rules in RULE, a set of data in MIDCONS and a set of applicable rules in $S A T$, i.e. $S A T:=p r S I E V E(R U L E, M I D C O N S)$. Denote by Conc $(r)$ the then-part of the rule $r$.

Put $S A T(r):=\operatorname{pr} S I E V E(R U L E, M I D C O N S \cup\{\operatorname{Conc}(r)\})$. So,

$$
h_{4}(r)=[\operatorname{Card}(S A T(r))-\operatorname{Card}(S A T)] / \operatorname{Card}(R U L E)
$$

(H5) Criterion H5 : This criterion expresses the fact that for the rule $r$ under consideration at moment $k$, if we use $r$, it is possible that the reasoning algorithm does not stop.

$$
h_{5}(r)= \begin{cases}1 & \text { if the condition }\left(^{*}\right) \text { is true; } \\ 0 & \text { if not. }\end{cases}
$$

Condition $\left(^{*}\right)$ : "The linguistic variable of $\operatorname{Conc}(r)$ occurs in $\mathcal{C}$ or in the if-part of one of the rules in $R U L E$ ". Then, the decision criterion for prCHOOSE(SAT,Criterion) is as follows: The rule $r$ in $S A T$ will be chosen if $h(r)$ is the is greatest among the rules in $S A T$, where $h(r)$ indicates the possibility degree the procedure can reach certain conclusions and is defined as follows:

$$
h(r)=\frac{1}{5} \sum_{i=1}^{5} w_{i} h_{i}(r) .
$$

\section{EXPERIMENT RESULTS}

We have constructed an expert system based on our reasoning algorithm presented above. With the co-operation with some high experienced medical experts in this field we established about 70 rules and collected more than 200 patient archives for our experiments. The parameters for the experiments is the following:

+ The sets of hedges: 
$H^{-}=$little (or less), relatively (or rather or more - or -less)\}, where little > relatively, $H^{+}=\{$many (or much), very $\}$, where much < very; and their fuzziness degrees are as follows: $\theta=0.628 ; \mu($ little $)=0.5024, \mu($ relatively $)=0.1256, \mu($ much $)=0.0744, \mu($ very $)=0.2976$.

+ The weights for computing the heuristic criteria $h(r)$ are: $w_{1}=0.92, w_{2}=0.85, w_{3}=0.5, w_{4}=0.6, w_{5}=1.0$.

+ The aggregation operator $\alpha$ for aggregating the component values $\nu_{X_{1}}\left(A_{01}\right), \nu X_{2}\left(A_{02}\right), \ldots, \nu X_{m}\left(A_{0 m}\right)$ is the average, i.e.

$$
\alpha\left(\nu_{X_{1}}\left(A_{01}\right), \nu_{X_{2}}\left(A_{02}\right), \ldots, \nu_{X_{m}}\left(A_{0 m}\right)\right)=\left(\sum_{j} \nu_{X_{j}}\left(A_{i j}\right)\right) / m .
$$

We give here the results of the reasoning algorithm for the real data of five hepatitis patients of Army Central Hospital at Hanoi, which were chosen without prior definitive intention. The data of these patients are given in Table 3.

\section{Table 3}

\begin{tabular}{|c|c|c|c|c|c|}
\hline Symptons & Patient A & Patient B & Patient C & Patient D & Patient E \\
\hline Age & 37 & 8 & 33 & 16 & 45 \\
\hline Body_status & ill & Rather_ill & $\begin{array}{l}\text { Very } \\
\text { Rather ill }\end{array}$ & Ill & Ill \\
\hline $\begin{array}{l}\text { Period_- } \\
\text { Temperature }\end{array}$ & 10 days & $-\cdots$ & $-\cdots$ & 10 days & 10 days \\
\hline $\begin{array}{l}\text { Temperature } \\
\text { degree }\end{array}$ & Little hot & Little_hot & $\begin{array}{l}\text { Very } \\
\text { littlte hot }\end{array}$ & Little hot & Little hot \\
\hline Sore_throat & $\begin{array}{l}-\ldots \text { (no } \\
\text { message) }\end{array}$ & Positive & $-{ }_{-1}$ & - - & $-{ }^{2}$ \\
\hline Nose sympton & $-\cdots$ & $-\cdots$ & running & $-\cdots$ & $-\cdots$ \\
\hline Head_paint & Aching & -- & $-\cdots$ & Aching & Aching \\
\hline Body_paint & Tire & Tire & Tire & Tire & Tire \\
\hline Skin_colour & Very_yellow & Yellow & Yellow & Yellow & Yellow \\
\hline $\begin{array}{l}\text { Eye_mucous_ } \\
\text { membrane }\end{array}$ & Yellow & Yellow & Yellow & Yellow & Yellow \\
\hline Fond_of_eating & Satiated & Satiated & Satiated & Satiated & Satiated \\
\hline Eat_fat & Afraid & Afraid & Afraid & Afraid & Afraid \\
\hline Sick_feelling & Positive & Positive & Positive & $-\cdots$ & $-\cdots$ \\
\hline Hbs_Ag_test & Very_positive & Negative & Positive & Positive & Positive \\
\hline SGOT_test & $\begin{array}{l}\text { Much } \\
\text { increasing }\end{array}$ & $\begin{array}{l}\text { Much } \\
\text { increasing }\end{array}$ & $\begin{array}{l}\text { Much } \\
\text { increasing }\end{array}$ & Increasing & $\begin{array}{l}\text { Much } \\
\text { increasing }\end{array}$ \\
\hline SGPT_test & $\begin{array}{l}\text { Much } \\
\text { increasing }\end{array}$ & $\begin{array}{l}\text { Much } \\
\text { increasing }\end{array}$ & $\begin{array}{l}\text { Much } \\
\text { increasing }\end{array}$ & $\begin{array}{l}\text { Much } \\
\text { increasing }\end{array}$ & $\begin{array}{l}\text { Much } \\
\text { increasing }\end{array}$ \\
\hline Bilirubin_test & $\begin{array}{l}\text { Much } \\
\text { increasing }\end{array}$ & Increasing & Increasing & Increasing & Increasing \\
\hline
\end{tabular}

Results of the diagnosis of the reasoning algorithm (Here Vi - He means riral hepatitis). 
Table 4

\begin{tabular}{|l|l|l|l|l|}
\hline Patient & Diagnosed disease & $\begin{array}{l}\text { Number of } \\
\text { inference steps }\end{array}$ & $\begin{array}{l}\text { Truth linguistic } \\
\text { value }\end{array}$ & $\begin{array}{l}\text { Values of } \\
\text { mapping v }\end{array}$ \\
\hline 5330 & Vi-He & 8 & Less rather much true & 0.87617 \\
\hline 2826 & $\mathrm{Vi}-\mathrm{He}$ & 9 & Very little much true & 0.86315 \\
\hline 8236 & $\mathrm{Vi}-\mathrm{He}$ & 11 & Little little much true & 0.87292 \\
\hline 1905 & $\mathrm{Vi}-\mathrm{He}$ & 11 & true & 0.86161 \\
\hline 105 & $\mathrm{Vi}-\mathrm{He}$ & 11 & Very little much true & 0.86315 \\
\hline
\end{tabular}

\section{CONCLUSIONS}

We have shown the applicability of hedge algebras in building a reasoning mechanism of a medicine expert system - a very sophisticated problem in general. The method seems to be rather simple and because the main idea of the interpolative method of reasoning based upon quantified semantics of terms is very closed to our intuition in reality, it is also much more suggestive. Although the practical validity of the method depends on the size of the experiment, the results of the algorithm which are much agreeable to the conclusions of the doctors in five chosen cases for experiment show that the method is applicable. Note that these cases are chosen randomly from 40 patients archives provided by the Army Central Hospital so that they have rather enough data for firing our established rules.

To improve the results, we are intending to deal with the following questions:

1) To expand the knowledge base (a set of rules) enough for making experiments: We have collected only about 70 rules. The difficulty is that the doctors are very difficult to formulate their knowledge in term of rules in general. Therefore, the collected rules may be not very appropriate to the considered application and we find that in order to gather additional knowledge in term of rules we need much more efforts.

2) To study more deeply those factors, which impact the efficiency of the reasoning method. In the paper we have not examined the influence of such factors yet. The factors may be:

+ Choose more suitable fuzziness measure of hedges and linguistic terms;

+ Choose a suitable quantified semantics mapping of linguistic domains $\operatorname{Dom}\left(X_{i}\right)$. The above examination we have assumed that all linguistic variables have the same quantified semantics mapping. In general they may be different each from the other ones.

+ Choose suitable aggregation operators: We have assumed that the aggregation operator is the simple average.

And so on.

Inspire of existing these not-yet-improved questions, it still shows that the method has many advantages:

+ The method is simple, unified and of much more intuitiveness.

+ Using quantified semantics mappings it is easy (with a simple algorithm) to solve the problem of linguistic approximation.

+ With several parameters of obvious suggestiveness it is also flexible to adapt specific applications.

\section{REFERENCES}

[1] A. Kaufmann, Introduction to the Theory of Fuzzy Subsets Vol. I, New York, San Francisco, London, 1975.

[2] E. H. Mamdani, Application of fuzzy logic to approximate reasoning using linguistic synthesis, IEEE Transaction on Computer 26 (1977) 1182-1191. 
[3] G. Resconi et al., Field theory and Modal Logic by Semantic Field to Make Uncertainty Emerge from Information, Int. Journal of General System 29 (2000) 737-782.

[4] J. B. Kiszka, M. E. Kochanska, D. S. Sliwinska, The inference of some fuzzy implication operators on the accuracy of a fuzzy model, part I, Fuzzy Sets and Systems 15 (1985) 111-128.

[5] J. B.Kiszka, M. E. Kochanska, D. S. Sliwinska, The inference of some fuzzy implication operators on the accuracy of a fuzzy model, part II, Fuzzy Sets and Systems 15 (1985) 223-240.

[6] L.T. Koczy and K. Hirota, Interpolative reasoning with insufficient evidence in sparse fuzzy rules bases, Inform. Sci. 71 (1993) 169-201.

[7] L.T. Koczy and K. Hirota, Approximate reasoning by linear rule interpolation and general approximation, Int. J. Approx. Reason. 9 (1993) 197-225.

[8] M. Mizumoto, Extended fuzzy reasoning, Approximate reasoning in expert systems (Ed, by M .M. Gupta, A. Kandel, W. Bandler, J. B. Kiszka), Elsevier Science Publishers B, V, (North-Holland), 1985.

[9] M. Mizumoto, Fuzzy inference with "if ... then ... else ..." under new compositional rules of inference, Management Decision Support Systems Using Fuzzy Sets and Possibility Theory (Ed. by Kacprzyk, R. Yager), ISR 83 Verlag TUV Rheinland GmbH, Koln, 1983.

[10] M. Mizumoto, H. J. Zimmermann, Comparison of fuzzy reasoning methods, Fuzzy Sets and Systems 18 (1982) 253-283.

[11] Nguyen Cat Ho, A consideration of some factors influencing on the accuracy of fuzzy conditional reasoning, Proceeding of Artificial Intelligence and Information-Control System of Robots conference of Slovak (AIICSR 97 Conf.) Bratislava, Sep 10-14, 1997.

[12] Nguyen Cat Ho, H. V. Nam, T. D. Khang, and N. H. Chau, Hedge algebras, Linguistic-valued Logic and Their Application to Fuzzy Reasoning, Internat. J. of Uncertainty, Fuzziness and Knowledge-Based Systems 7 (4) (1999).

[13] Nguyen Cat Ho, Huynh Van Nam, Ordered Structure-Based Semantics of Linguistic Terms of Linguistic Variables and Approximate Reasoning, AIP conference proceedings on Computing Anticipatory Systems, CASYS'99 Third International Conference, 98-116.

[14] Nguyen Cat Ho, Tran Thai Son, On fuzzy model error, Journal of Informatics and Cybernetics 13 (1) (1997) 66-72.

[15] Nguyen Cat Ho, Tran Thai Son, Fuzzy logic and fuzzy decision based on ordered structure of linguistic values, Journal of Informatics and Cybernetics 9 (4) (1993) 1-9.

[16] Nguyen Cat Ho, Tran Thai Son, On the distance between values of linguistic variables in hedge algebra, Journal of Computer Science and Cybernetics 11 (1) (1995) 10-20.

[17] Nguyen Cat Ho, W. Wechler, Hedge algebra: An algebraic approach to structures of sets of linguistic truth values, Fuzzy Sets and Systems 35 (1990) 281-293.

[18] Nguyen Cat Ho, W. Wechler, Extended algebra and their application to fuzzy logic, Fuzzy sets and systems $\mathbf{5 2}$ (1992) 259-281.

[19] Nguyen Hai Chau, "Some problems in designing a specific computer network and network chains problem with fuzzy reasoning technique," PhD Dissertations (in Vietnamese)

[20] Resconi et al., Transformations of Fuzzy Sets by Modal Logic (Hedge Algebra ), in preparation for publication.

[21] R.R. Yager and A. Rybakov, Noncommutative self-identity aggregation, Fuzzy Sets and Systems 85 (1997) $73-82$.

[22] R.R. Yager, Aggregation operators and fuzzy systems modelling, Fuzzy Sets and Systems 67 (1994) $129-145$.

[23] Tran Dinh Khang, The construction of measure function in hedge algebra and its application in linguistic reasoning, Journal of Informatics and Cybernetics 13 (1) (1997).

[24] W.H. Hsiao, S.M. Chen, C.H. Lee, A New Interpolative Reasoning Method in Sparse Rule-Based Systems, Volume 93, (1) (1998).

[25] Z. Cao, A. Kandel, Applicability of some fuzzy implication operators, Fuzzy sets and systems 31 (1989) 151-186. 
[26] Y. Shi, M. Mizumoto, Reasoning conditions on Koczy's interpolative reasoning method in sparse fuzzy rule bases, Part II, Fuzzy sets and systems 87 (1997) 47-56.

[27] Y. Shi, M. Mizumoto, A note on reasoning conditions of Kocy's interpolative reasoning method (Short Communication), Fuzzy Sets and Systems 96 (1998) 373-379.

[28] Y. Shi, M. Mizumoto, and Z.Q. Wu, Reasoning conditions on Koczy's interpolative reasoning methods in sparse fuzzy rules bases, Fuzzy Sets and Systems 75 (1995) 63-71.

Received January 15, 2002

Tran Dinh Khang - Ha Noi Technology University

Le Xuan Viet - Quy Nhon Pedagogical University

Nguyen Cat Ho, Tran Thai Son - Institute of Information Technology 